\title{
INVESTIGATION OF G-NETWORK WITH RANDOM DELAY OF SIGNALS AT NONSTATIONARY BEHAVIOUR
}

\author{
Mikhail Matalytski ${ }^{1}$, Victor Naumenko ${ }^{2}$ \\ ${ }^{I}$ Institute of Mathematics, Czestochowa University of Technology \\ Czestochowa, Poland \\ ${ }^{2}$ Faculty of Mathematics and Computer Science, Grodno State University \\ Grodno, Belarus \\ Im.matalytski@gmail.com, 2victornn86@gmail.com
}

\begin{abstract}
The object of this research is an open queueing G-network with signals with random delay. The purpose of the research is investigation of such a network at the transient behavior. It is considered the case when the intensity of the incoming flow of positive and negative messages and service intensity of messages do not depend on time. All the systems in the network are one-line. It is described the model of computer system DDoS-attacks, the effect of penetration of the virus in a computer network in the form of G-network with random delay of signals. Approximate expressions are obtained for the time-dependent probabilities of states and the average characteristics of the network. Examples are calculated.
\end{abstract}

Keywords: G-network, negative messages, the transient behavior, signals with random delay, DDoS-attack

\section{Introduction}

Previously [1] G-networks with signals were considered (which it could be either negative messages or triggers), the effect of which was manifested immediately, i.e. activation time of any signal equal to zero and therefore not taken into account in the analysis of such networks. Now we assume that entering the queueing system (QS), signal is activated not immediately, but only after a random time. It should be noted that such a network with a one-line QS, at some generalized assumptions about the service time of positive customers, was discussed in [2], the stationary network state probabilities of the states were found in the multiplicative form.

This assumption can be used for modeling real information and telecommunications networks [3]. Negative messages and signals in the model can describe viruses in the systems that start operating after a random time, while the signal "corrected" - becomes a positive customer or "brings destruction" (i.e. becoming a negative message), destroys the positive customer in the system (or "recognized") or "destroyed" - not rendering any impact on the system. 


\section{Network description, formulation of the problem}

Consider an open G-network with $n$ one-line QS $[4,5]$. In QS $S_{i}$ come from outside (system $S_{0}$ ) independent Poisson flow of positive messages with intensity $\lambda_{0 i}^{+}$and Poisson flow of signals with intensity $\lambda_{0 i}^{-}, i=\overline{1, n}$. The probability that the positive application gets serviced in $S_{i}$ during time $[t, t+\Delta t)$, if at the current time moment $t$ in the system there is $k$ messages, is equal to $\mu_{i}^{+}(k) \Delta t+o(\Delta t)$. Positive message serviced in $S_{i}$ with probability $p_{i j}^{+}$comes in QS $S_{j}$ as a positive message, and with probability $p_{i j}^{-}$- as a signal, and with probability $p_{i 0}=1-\sum_{j=1}^{n}\left(p_{i j}^{+}+p_{i j}^{-}\right)$ comes out from the network to external environment, $i, j=\overline{1, n}$.

Each incoming signal is activated for some random time interval. The probability that the incoming in the QS $S_{i}$ signal is activated during time $[t, t+\Delta t)$, on condition that, in it QS at moment time $t$ there are $l$ nonactivated signals, is equal to $\mu_{i}^{-}(l) \Delta t+o(\Delta t)$. After that time (activation time):

- with probability $q_{i j}^{+}$the signal is activated as a trigger, moving one positive message of QS $S_{i}$ to QS $S_{j}$, while its message remains positive;

- with probability $q_{i j}^{-}$the signal is activated again as a trigger, moving one positive message of QS $S_{i}$ to QS $S_{j}$, but thus its message in QS $S_{j}$ becomes a signal;

- with probability $q_{i 0}=1-\sum_{j=1}^{n}\left(q_{i j}^{+}+q_{i j}^{-}\right)$signal is triggered by a negative message, that destroying one positive message in QS $S_{i}$ and leaves the network.

The message, which moves from QS $S_{i}$ to QS $S_{j}$ (as a positive message or signal), terminates its service in the QS $S_{i}$. If after the activation signal the system is missing positive messages, then the signal comes out from the network without affecting the functioning of the network as a whole.

The network state at the moment time $t$ will be vector $k(t)=(k, l, t)=\left(\left(k_{1}, l_{1}, t\right),\left(k_{2}, l_{2}, t\right), \ldots,\left(k_{n}, l_{n}, t\right)\right)$, which forms a homogeneous Markov process with a countable number of states, where the state $\left(k_{i}, l_{i}, t\right)$ means, that at moment time $t$ in QS $S_{i}$ there are $k_{i}$ positive messages and $l_{i}$ nonactivated signals, $i=\overline{1, n}$. We introduce the vectors $(k, t)=\left(k_{1}, k_{2}, \ldots, k_{n}, t\right)$ and $(l, t)=\left(l_{1}, l_{2}, \ldots, l_{n}, t\right), I_{i}$ - vector, which is $i$-th component is equal to 1 , all the others are $0, i=\overline{1, n}$. 
It is required to find time-dependent state probabilities and average characteristics of the considered network.

Lemma. [4] Let $P(k, l, t)$ - state probability $(k, l)$ at moment time $t$. State probabilities of the considered network satisfy system of difference-differential equations (DDE):

$$
\begin{aligned}
& \frac{d P(k, l, t)}{d t}=-\sum_{i=1}^{n}\left[\lambda_{0 i}^{+}+\lambda_{0 i}^{+}+\mu_{i}^{+}\left(k_{i}\right)\left(1-p_{i i}^{+}\right)+\mu_{i}^{-}\left(l_{i}\right)\right] P(k, l, t)+ \\
& +\sum_{i=1}^{n} \lambda_{0 i}^{+} u\left(k_{i}\right) P\left(k-I_{i}, l, t\right)+\sum_{i=1}^{n} \lambda_{0 i}^{-} u\left(l_{i}\right) P\left(k, l-I_{i}, t\right)+ \\
& +\sum_{i=1}^{n} \mu_{i}^{+}\left(k_{i}+1\right) p_{i 0} P\left(k+I_{i}, l, t\right)+\sum_{i=1}^{n} \mu_{i}^{-}\left(l_{i}+1\right) q_{i 0} P\left(k+I_{i}, l+I_{i}, t\right)+ \\
& +\sum_{i=1}^{n} \mu_{i}^{-}\left(l_{i}+1\right)\left(1-u\left(k_{i}\right)\right) P\left(k, l+I_{i}, t\right)+\sum_{i=1}^{n} \sum_{\substack{j=1 \\
j \neq i}}^{n} \mu_{i}^{+}\left(k_{i}+1\right) u\left(k_{j}\right) p_{i j}^{+} P\left(k+I_{i}-I_{j}, l, t\right)+ \\
& +\sum_{i=1}^{n} \sum_{j=1}^{n} \mu_{i}^{+}\left(k_{i}+1\right) u\left(l_{j}\right) p_{i j}^{-} P\left(k+I_{i}, l-I_{j}, t\right)+ \\
& +\sum_{i=1}^{n} \sum_{j=1}^{n} \mu_{i}^{-}\left(l_{i}+1\right) u\left(k_{j}\right) q_{i j}^{+} P\left(k+I_{i}-I_{j}, l+I_{i}, t\right)+ \\
& +\sum_{i=1}^{n} \sum_{\substack{j=1 \\
j \neq i}}^{n} \mu_{i}^{-}\left(l_{i}+1\right) u\left(l_{j}\right) q_{i j}^{-} P\left(k+I_{i}, l+I_{i}-I_{j}, t\right)+\sum_{i=1}^{n} \mu_{i}^{-}\left(l_{i}\right) q_{i i}^{-} P\left(k+I_{i}, l, t\right),
\end{aligned}
$$

where $\mu_{i}^{+}(0)=0, \mu_{i}^{-}(0)=0, u(x)=\left\{\begin{array}{l}1, x>0 \\ 0, x \leq 0\end{array}\right.$ - Heaviside function.

\section{Attack model on the computer system, virus penetration effect into the computer network}

Damage from the virus penetration into the computer network may be quite different, from a slight increase in the size of outgoing traffic to complete failure of the network or loss of vital information. If the target of the attack is to destroy or steal information, the damage from a successful attack is equal to the value of this information. In case of theft of information - especially in cases of deliberate attacks on obviously some sacrifice - the results can be disastrous for the owner of the data, especially if we are talking about the leak of information critical to a company, organization or even a state. Customer databases, financial and technical documents, bank account numbers, details of offers - the list is endless [6]. 
The failure of computers and networks, or a sharp slowdown in their work is deliberate or accidental. In the case of a deliberate attack by a virus or a Trojan or the destruction of critical elements of the system, which causes an inoperable or overloaded network DDoS (Distributed Denial of Service) attack, or even in any way affects the efficiency of the system [7].

Among computer intruders DDoS attacks are growing in popularity [8]. Attack purpose is to block the legitimate users access to a website, or to make it extremely difficult. As a result of the attack there are broken or blocked completely legitimate service users, networks, systems and other resources. It uses two types of effects: the attack on the communication channel, which "hammered" a huge mass of specially crafted requests and results in an overflow system or network using a huge amount of information that can not be processed (in this case the network is operating under high load), or use of holes in the software and network protocols to block customers' access to resources information system, in this case, the whole system stops working or the network does as a result of the system sending the data packets that it does not expect, and this leads to a system stop or restarts it [8].

Despite the fact that DDoS-attack can be directed to a separate PC, today this method of network attack is used mainly against government and corporate resources (a well-protected large company or government agency), for which there are several reasons. Network DDoS-attack types by using a botnet (zombie networks) - a large number of special malware infected computers that commanded from the control center (from the attacker) start sending many special requests to the target computer, blocking access to legitimate users $[9,10]$.

\section{DDoS-attack model on a computer network in the form of G-networks with signals with random delay}

The model considered above, an attack on a computer network, can be described as an open G-network with signals that are activated after a random time.

In this case, under the positive messages or simply messages, it will be involved requests from different computers. Under negative messages we will understand the data or packets that the system does not expect (the virus program), which leads it to stop or restarts it. In other words, such requests destroy other messages in the system.

Selected nodes are attacked and the attacker gets their admin rights. For each of the captured nodes there are installed Trojans that run in the background. Under the signals in the network we will mean such programs, which are then activated by a random time in the attacker team. They are called zombie computers and their users are not even aware that they are potential participants in DDoS-attack. This means that at any point in time in these hosts there is at least one activated signal.

Next, the attacker sends certain commands to captured computers and those in turn carry out a powerful DDoS-attack on the target computer. 


\section{Finding state probabilities of the QS network}

We will assume that all queuing network systems are one-line, and messages service duration in the QS has an exponential distribution with parameter $\mu_{i}^{+}$. Consequently, in this case $\mu_{i}^{+}\left(k_{i}\right)=u\left(k_{i}\right) \mu_{i}^{+}, i=\overline{1, n}$.

Denote by $\Psi_{2 n}(z, t)$, where $z=\left(z_{1}, z_{2}, \ldots, z_{n}, z_{n+1}, \ldots, z_{2 n}\right)$, generating function of the dimension of $2 n$ :

$$
\begin{gathered}
\Psi_{2 n}(z, t)=\sum_{k_{1}=0}^{\infty} \ldots \sum_{k_{n}=0}^{\infty} \sum_{l_{1}=0}^{\infty} \ldots \sum_{l_{n}=0}^{\infty} P\left(z_{1}, z_{2}, \ldots, z_{n}, z_{n+1,} \ldots, z_{2 n}\right) z_{1}^{k_{1}} \ldots z_{n}^{k_{n}} z_{n+1}^{l_{1}} \ldots z_{2 n}^{l_{n}}= \\
=\sum_{k_{1}=0}^{\infty} \ldots \sum_{k_{n}=0}^{\infty} \sum_{l_{1}=0}^{\infty} \ldots \sum_{l_{n}=0}^{\infty} P(k, l, t) \prod_{i=1}^{n} z_{i}^{k_{i}} z_{n+i}^{l_{i}},|z|<1
\end{gathered}
$$

the summation is taking for each $k_{i}, l_{i}$ from 0 to $\infty, i=\overline{1, n}$.

According to the described DDoS-attack model of our network, find that all the systems in the network operate in high load. We will also assume that $k_{i}(t)>0$ $\forall t>0, i=\overline{1, n}$. Furthermore, according to the same model, we will assume that $l_{i}(t)>0 \quad \forall t>0$. Multiplying each of the equations (1) to $\prod_{m=1}^{n} z_{m}^{k_{m}} z_{m}^{l_{m}}$ and summing over all possible values $k_{m}$ and $l_{m}$ from 1 to $+\infty, m=\overline{1, n}$.

Converting the sums included in the right part of relations (2), we will obtain a homogeneous linear differential equation [4]:

$$
\begin{gathered}
\frac{d \Psi_{2 n}(z, t)}{d t}=-\sum_{i=1}^{n}\left[\lambda_{0 i}^{+}+\lambda_{0 i}^{+}+\mu_{i}^{+}\left(1-p_{i i}^{+}\right)+\mu_{i}^{-}-\lambda_{0 i}^{+} z_{i}-\lambda_{0 i}^{-} z_{n+i}-\mu_{i}^{-} \frac{q_{i 0}}{z_{i} z_{n+i}}-\mu_{i}^{-} \frac{q_{i i}^{-}}{z_{i}}-\right. \\
\left.-\sum_{j=1}^{n}\left(\mu_{i}^{+} p_{i j}^{+} \frac{z_{j}}{z_{i}}+\mu_{i}^{+} p_{i j}^{-} \frac{z_{n+j}}{z_{i}}+\mu_{i}^{-} q_{i j}^{+} \frac{z_{j}}{z_{i} z_{n+i}}+\mu_{i}^{-} q_{i j}^{-} \frac{z_{n+j}}{z_{i} z_{n+i}}\right)\right] \Psi_{2 n}(z, t) .
\end{gathered}
$$

Its solution has the form

$$
\begin{gathered}
\Psi_{n}(z, t)=C_{n} \exp \left\{-\sum_{i=1}^{n}\left[\lambda_{0 i}^{+}+\lambda_{0 i}^{+}+\mu_{i}^{+}\left(1-p_{i i}^{+}\right)+\mu_{i}^{-}-\lambda_{0 i}^{+} z_{i}-\lambda_{0 i}^{-} z_{n+i}-\mu_{i}^{-} \frac{q_{i 0}}{z_{i} z_{n+i}}-\right.\right. \\
\left.\left.-\mu_{i}^{-} \frac{q_{i i}^{-}}{z_{i}}-\sum_{j=1}^{n}\left(\mu_{i}^{+} p_{i j}^{+} \frac{z_{j}}{z_{i}}+\mu_{i}^{+} p_{i j}^{-} \frac{z_{n+j}}{z_{i}}+\mu_{i}^{-} q_{i j}^{+} \frac{z_{j}}{z_{i} z_{n+i}}+\mu_{i}^{-} q_{i j}^{-} \frac{z_{n+j}}{z_{i} z_{n+i}}\right)\right] t\right\} .
\end{gathered}
$$

We assume that at the initial time the network is able to state $\left(\alpha_{1}, \alpha_{2}, \ldots, \alpha_{2 n}, 0\right)$, $\alpha_{i}>0, \alpha_{n+i}>0, P\left(\alpha_{1}, \alpha_{2}, \ldots, \alpha_{2 n}, 0\right)=1, P\left(k_{1}, k_{2}, \ldots, k_{n}, l_{1}, l_{2}, \ldots, l_{n} 0\right)=0, \forall \alpha_{i} \neq k_{i}, l_{i}$, 
$i=\overline{1, n}$. Then the initial condition for the last equation will be $\Psi_{2 n}(z, 0)=P\left(\alpha_{1}, \alpha_{2}, \ldots, \alpha_{2 n}, 0\right) \prod_{m=1}^{n} z_{m}^{\alpha_{m}} z_{n+m}^{\alpha_{n+m}}=\prod_{m=1}^{n} z_{m}^{\alpha_{m}} z_{n+m}^{\alpha_{n+m}}$. Using it, we obtain $C_{n}=1$.

Theorem. If at the initial moment of time the queueing network is in a state $\left(\alpha_{1}, \alpha_{2}, \ldots, \alpha_{2 n}, 0\right), \alpha_{i}>0, \alpha_{n+i}>0, i=\overline{1, n}$, then the expression for the generating function $\Psi_{2 n}(z, t)$, then taking into account the decomposition of exponent into Maclaurin series, has the form

$$
\begin{aligned}
& \Psi_{2 n}(z, t)=a_{0}(t) \sum_{\substack{b_{j}=0 \\
j=1, n, j \neq i}}^{\infty} \sum_{\substack{c_{j}=0 \\
j=1, n, j \neq i}}^{\infty} \sum_{\substack{d_{j}=0 \\
j=1, n, j \neq i}}^{\infty} \sum_{\substack{g_{j}=0 \\
j=1, n, j \neq i}}^{\infty} x
\end{aligned}
$$

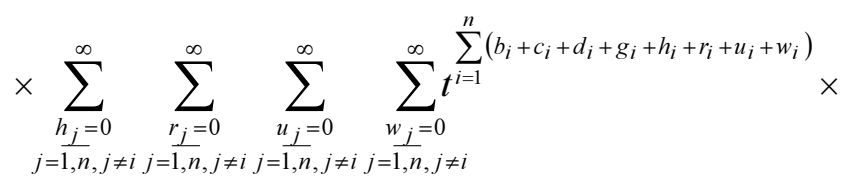

$$
\begin{aligned}
& \times \prod_{i=1}^{n}\left[\frac{\left(\lambda_{0 i}^{+}\right)^{b_{i}}\left(\lambda_{0 i}^{-}\right)^{c_{i}}\left(\mu_{i}^{+}\right)^{h_{i}+r_{i}}\left(\mu_{i}^{-}\right)^{d_{i}+g_{i}+u_{i}+w_{i}} q_{i 0}^{d_{i}+g_{i}}\left(q_{i i}^{-}\right)^{g_{i}}}{b_{i} ! c_{i} ! d_{i} ! g_{i} ! h_{i} ! r_{i} ! u_{i} ! w_{i} !} \times\right. \\
& \left.\times\left(\prod_{j=1}^{n} p_{i j}^{+}\right)^{h_{i}}\left(\prod_{j=1}^{n} p_{i j}^{-}\right)^{r_{i}}\left(\prod_{j=1}^{n} q_{i j}^{+}\right)^{u_{i}}\left(\prod_{j=1}^{n} q_{i j}^{-}\right)^{w_{i}} z_{i}^{\alpha_{i}+b_{i}-d_{i}-g_{i}+H-h_{i}-r_{i}+U-u_{i}-w_{i}} z_{n+i}^{\alpha_{n+i}+c_{i}-d_{i}+R-u_{i}+W-w_{i}}\right], \\
& \text { where } H=\sum_{i=1}^{n} h_{i}, R=\sum_{i=1}^{n} r_{i}, U=\sum_{i=1}^{n} u_{i}, W=\sum_{i=1}^{n} w_{i} \text {, } \\
& a_{0}(t)=\exp \left\{-\sum_{i=1}^{n}\left[\lambda_{0 i}^{+}+\lambda_{0 i}^{+}+\mu_{i}^{+}\left(1-p_{i i}^{+}\right)+\mu_{i}^{-}\right] t\right\} .
\end{aligned}
$$

Proof. This can be proved in a similar way as for networks with positive and negative messages at transition behaviour [5]. We have:

$$
\Psi_{n}(z, t)=a_{0}(t) \prod_{i=1}^{n} a_{i}(z, t) \prod_{m=1}^{n} z_{m}^{\alpha_{m}} z_{n+m}^{\alpha_{n+m}},
$$

where

$$
\begin{gathered}
a_{1}(z, t)=\exp \left\{t \sum_{i=1}^{n} \lambda_{0 i}^{+} z_{i}\right\}=\prod_{i=1}^{n} \exp \left\{\lambda_{0 i}^{+} t z_{i}\right\}=\prod_{i=1}^{n} \sum_{b_{i}=0}^{\infty} \frac{\left[\lambda_{0 i}^{+} t z_{i}\right]^{b_{i}}}{b_{i} !}=\sum_{b_{1}=0}^{\infty} \ldots \sum_{b_{n}=0}^{\infty} \prod_{i=1}^{n} \frac{\left[\lambda_{0 i}^{+} t z_{i}\right]^{b_{i}}}{b_{i} !}= \\
=\sum_{b_{1}=0}^{\infty} \ldots \sum_{b_{n}=0}^{\infty} \frac{t^{b_{1}+b_{2}+\ldots+b_{n}}}{b_{1} ! b_{2} ! \ldots \cdot b_{n} !}\left(\lambda_{01}^{+}\right)^{b_{1}} \cdot \ldots \cdot\left(\lambda_{0 n}^{+}\right)^{b_{n}} z_{1}^{b_{1}} \cdot \ldots \cdot z_{n}^{b_{n}}
\end{gathered}
$$




$$
\begin{aligned}
& a_{2}(z, t)=\exp \left\{t \sum_{i=1}^{n} \lambda_{0 i}^{-} z_{n+i}\right\}=\sum_{c_{1}=0}^{\infty} \ldots \sum_{c_{n}=0}^{\infty} \frac{t^{c_{1}+c_{2}+\ldots+c_{n}}}{c_{1} ! c_{2} ! \ldots \cdot c_{n} !}\left(\lambda_{01}^{-}\right)^{c_{1}} \cdot \ldots \cdot\left(\lambda_{0 n}^{-}\right)^{c_{n}} z_{n+1}^{c_{1}} \cdot \ldots \cdot z_{2 n}^{c_{n}}, \\
& a_{3}(z, t)=\exp \left\{t \sum_{i=1}^{n} \mu_{i}^{-} \frac{q_{i 0}}{z_{i} z_{n+i}}\right\}=\prod_{i=1}^{n} \exp \left\{\mu_{i}^{-} t \frac{q_{i 0}}{z_{i} z_{n+i}}\right\}=\sum_{d_{1}=0}^{\infty} \ldots \sum_{d_{n}=0}^{\infty} \prod_{i=1}^{n} \frac{\left[\mu_{i}^{-} q_{i 0} t z_{i}^{-1} z_{n+i}^{-1}\right]^{d_{i}}}{d_{i} !}= \\
& =\sum_{d_{1}=0}^{\infty} \ldots \sum_{d_{n}=0}^{\infty} \frac{t^{d_{1}+d_{2}+\ldots+d_{n}}}{d_{1} ! d_{2} ! \cdot \ldots \cdot d_{n} !}\left(\mu_{1}^{-} q_{10}\right)^{d_{1}} \cdot \ldots \cdot\left(\mu_{n}^{-} q_{n 0}\right)^{d_{n}} z_{1}^{-d_{1}} \cdot \ldots \cdot z_{n}^{-d_{n}} z_{n+1}^{-d_{1}} \cdot \ldots \cdot z_{2 n}^{-d_{n}}, \\
& a_{4}(z, t)=\exp \left\{t \sum_{i=1}^{n} \mu_{i}^{-} \frac{q_{i i}^{-}}{z_{i}}\right\}=\prod_{i=1}^{n} \exp \left\{\mu_{i}^{-} t \frac{q_{i i}^{-}}{z_{i}}\right\}=\sum_{g_{1}=0}^{\infty} \ldots \sum_{g_{n}=0}^{\infty} \prod_{i=1}^{n} \frac{\left[\mu_{i}^{-} q_{i i}^{-} t z_{i}^{-1}\right]^{g_{i}}}{g_{i} !}= \\
& =\sum_{g_{1}=0}^{\infty} \ldots \sum_{g_{n}=0}^{\infty} \frac{t^{g_{1}+g_{2}+\ldots+g_{n}}}{g_{1} ! g_{2} ! \ldots \cdot g_{n} !}\left(\mu_{1}^{-} q_{11}\right)^{g_{1}} \cdot \ldots \cdot\left(\mu_{n}^{-} q_{n n}\right)^{g_{n}} z_{1}^{-g_{1}} \cdot \ldots \cdot z_{n}^{-g_{n}}, \\
& a_{5}(z, t)=\exp \left\{\sum_{i, j=1}^{n} t \mu_{i}^{+} p_{i j}^{+} \frac{z_{j}}{z_{i}}\right\}=\prod_{i=1}^{n} \prod_{j=1}^{n} \exp \left\{t \mu_{i}^{+} p_{i j}^{+} \frac{z_{j}}{z_{i}}\right\}=\prod_{i=1}^{n} \prod_{j=1}^{n} \sum_{h_{i}=0}^{\infty} \frac{\left[t \mu_{i}^{+} p_{i j}^{+} z_{j} z_{i}^{-1}\right]^{h_{i}}}{h_{i} !}= \\
& =\sum_{h_{1}=0}^{\infty} \ldots \sum_{h_{n}=0}^{\infty} \prod_{i=1}^{n} \prod_{j=1}^{n} \frac{\left[t \mu_{i}^{+} p_{i j}^{+} z_{j} z_{i}^{-1}\right]^{h_{i}}}{h_{i} !}= \\
& =\sum_{h_{1}=0}^{\infty} \ldots \sum_{h_{n}=0}^{\infty} t^{h_{1}} \cdot \ldots \cdot t^{h_{n}} \frac{\left(\prod_{j=1}^{n} \mu_{1}^{+} p_{1 j}^{+}\right)^{h_{1}} \cdot \ldots \cdot\left(\prod_{j=1}^{n} \mu_{n}^{+} p_{n j}^{+}\right)^{h_{n}}}{h_{1} ! \ldots \cdot h_{n} !} z_{1}^{H-h_{1}} \cdot \ldots \cdot z_{n}^{H-h_{n}} \text {, } \\
& a_{6}(z, t)=\exp \left\{\sum_{i, j=1}^{n} t \mu_{i}^{+} p_{i j}^{-} \frac{z_{n+j}}{z_{i}}\right\}=\prod_{i=1}^{n} \prod_{j=1}^{n} \exp \left\{t \mu_{i}^{+} p_{i j}^{-} \frac{z_{n+j}}{z_{i}}\right\}=\prod_{i=1}^{n} \prod_{j=1}^{n} \sum_{r_{i}=0}^{\infty} \frac{\left[t \mu_{i}^{+} p_{i j}^{-} z_{n+j} z_{i}^{-1}\right]^{r_{i}}}{r_{i} !}= \\
& =\sum_{r_{1}=0}^{\infty} \ldots \sum_{r_{n}=0}^{\infty} \prod_{i=1}^{n} \prod_{j=1}^{n} \frac{\left[t \mu_{i}^{+} p_{i j}^{-} z_{n+j} z_{i}^{-1}\right]^{r_{i}}}{r_{i} !}=\sum_{r_{1}=0}^{\infty} \ldots \sum_{r_{n}=0}^{\infty} t^{r_{1}} \cdot \ldots \cdot t^{r_{n}} \frac{\left(\prod_{j=1}^{n} \mu_{1}^{+} p_{1 j}^{-}\right)^{r_{1}} \cdot \ldots \cdot\left(\prod_{j=1}^{n} \mu_{n}^{+} p_{n j}^{-}\right)^{r_{n}}}{r_{1} ! \ldots \cdot r_{n} !} \times \\
& \times z_{1}^{-r_{1}} z_{2}^{-r_{2}} \ldots z_{n}^{-r_{n}} z_{n+1}^{r_{1}+r_{2}+\ldots+r_{n}} z_{n+2}^{r_{1}+r_{2}+\ldots+r_{n}} \cdot \ldots \cdot z_{2 n}^{r_{1}+r_{2}+\ldots+r_{n}} \\
& a_{7}(z, t)=\exp \left\{\sum_{i, j=1}^{n} t \mu_{i}^{-} q_{i j}^{+} \frac{z_{j}}{z_{i} z_{n+i}}\right\}=\prod_{i=1}^{n} \prod_{j=1}^{n} \exp \left\{t \mu_{i}^{-} q_{i j}^{+} \frac{z_{j}}{z_{i} z_{n+i}}\right\}= \\
& =\sum_{u_{1}=0}^{\infty} \ldots \sum_{u_{n}=0}^{\infty} \prod_{i=1}^{n} \prod_{j=1}^{n} \frac{\left[t \mu_{i}^{-} q_{i j}^{+} z_{j} z_{i}^{-1} z_{n+i}^{-1}\right]^{u_{i}}}{u_{i} !}= \\
& =\sum_{u_{1}=0}^{\infty} \ldots \sum_{u_{n}=0}^{\infty} t^{u_{1}} \cdot \ldots \cdot t^{u_{n}} \frac{\left(\prod_{j=1}^{n} \mu_{1}^{-} q_{1 j}^{+}\right)^{u_{1}} \cdot \ldots \cdot\left(\prod_{j=1}^{n} \mu_{n}^{-} q_{n j}^{+}\right)^{u_{n}}}{u_{1} ! \ldots \cdot u_{n} !} z_{1}^{U-u_{1}} z_{2}^{U-u_{2}} \ldots z_{n}^{U-u_{n}} z_{n+1}^{-u_{1}} z_{n+2}^{-u_{2}} \cdot \ldots \cdot z_{2 n}^{-u_{n}} \text {, }
\end{aligned}
$$




$$
\begin{aligned}
& a_{8}(z, t)=\exp \left\{\sum_{i, j=1}^{n} t \mu_{i}^{-} q_{i j}^{-} \frac{z_{n+j}}{z_{i} z_{n+i}}\right\}=\prod_{i=1}^{n} \prod_{j=1}^{n} \exp \left\{t \mu_{i}^{-} q_{i j}^{-} \frac{z_{n+j}}{z_{i} z_{n+i}}\right\}= \\
& =\sum_{w_{1}=0}^{\infty} \ldots \sum_{w_{n}=0}^{\infty} \prod_{i=1}^{n} \prod_{j=1}^{n} \frac{\left[t \mu_{i}^{-} q_{i j}^{-} z_{n+j} z_{i}^{-1} z_{n+i}^{-1}\right]^{w_{i}}}{w_{i} !}= \\
& =\sum_{w_{1}=0}^{\infty} \ldots \sum_{w_{n}=0}^{\infty} t^{w_{1}} \cdot \ldots \cdot t^{w_{n}} \frac{\left(\prod_{j=1}^{n} \mu_{1}^{-} q_{1 j}^{-}\right)^{w_{1}} \cdot \ldots \cdot\left(\prod_{j=1}^{n} \mu_{n}^{-} q_{n j}^{-}\right)^{w_{n}}}{w_{1} ! \ldots \cdot w_{n} !} z_{1}^{-w_{1}} z_{2}^{-w_{2}} \ldots z_{n}^{-w_{n}} z_{n+1}^{W-w_{1}} z_{n+2}^{W-w_{2}} \cdot \ldots \cdot z_{2 n}^{W-w_{n}} .
\end{aligned}
$$

Multiplying $a_{0}(t), a_{i}(z, t)$, and $\prod_{m=1}^{n} z_{m}^{\alpha_{m}} z_{n+m}^{\alpha_{n+m}}$ we will obtain an expression (3), $i=\overline{1,8}$.

State probability of $P\left(k_{1}, k_{2}, . ., k_{n}, l_{1}, l_{2}, \ldots, l_{n}, t\right)$ is the coefficient of $z_{1}^{k_{1}} z_{2}^{k_{2}}, \ldots, z_{n}^{k_{n}} z_{n+1}^{l_{1}}, z_{n+2}^{l_{2}}, \ldots, z_{2 n}^{l_{n}}$ in the expansion of $\Psi_{2 n}(z, t)$ in multiple series (3), with the proviso that at the initial time the network is in a state $\left(\alpha_{1}, \alpha_{2}, \ldots, \alpha_{2 n}, 0\right)$.

\section{Finding the average characteristics}

As it is known, with the help of the generating function, a different average of network characteristics can be found in the transient behaviour. For example, for the average number of positive messages in the network system $S_{x}$ will be used the relation:

$$
\begin{aligned}
N_{x}(t) & =\left.\frac{\partial \Psi_{2 n}(z, t)}{\partial z_{x}}\right|_{z=(1,1, \ldots, 1)}=a_{0}(t) \sum_{\substack{b_{j}=0 \\
j=1, n, j \neq i}}^{\infty} \sum_{\substack{c_{j}=0 \\
j=1, n, j \neq i}}^{\infty} \sum_{\substack{d_{j}=0 \\
j=1, n, j \neq i}}^{\infty} \sum_{\substack{g_{j}=0 \\
j=1, n, j \neq i}}^{\infty} \times \\
\times & \sum_{\substack{h_{j}=0 \\
j=1, n, j \neq i}}^{\infty} \sum_{\substack{r_{j}=0 \\
j=1, n, j \neq i}}^{\infty} \sum_{\substack{u_{j}=0 \\
j=1, n, j \neq i}}^{\infty} \sum_{\substack{w_{j}=0 \\
\sum_{j}=1, n, j \neq i}}^{\infty} \sum^{\sum_{i=1}\left(b_{i}+c_{i}+d_{i}+g_{i}+h_{i}+r_{i}+u_{i}+w_{i}\right)} \times \\
& \times\left(\alpha_{x}+b_{x}-d_{x}-g_{x}+H-h_{x}-r_{x}+U-u_{x}-w_{x}\right) \times \\
& \times \prod_{i=1}^{n}\left[\frac{\left(\lambda_{0 i}^{+}\right)^{b_{i}}\left(\lambda_{0 i}^{-}\right)^{c_{i}}\left(\mu_{i}^{+}\right)^{h_{i}+r_{i}}\left(\mu_{i}^{-}\right)^{d_{i}+g_{i}+u_{i}+w_{i}} q_{i 0}^{d_{i}+g_{i}}\left(q_{i i}^{-}\right)^{g_{i}}}{b_{i} ! c_{i} ! d_{i} ! g_{i} ! h_{i} ! r_{i} ! u_{i} ! w_{i} !} \times\right. \\
& \left.\times\left(\prod_{j=1}^{n} p_{i j}^{+}\right)^{h_{i}}\left(\prod_{j=1}^{n} p_{i j}^{-}\right)^{r_{i}}\left(\prod_{j=1}^{n} q_{i j}^{+}\right)^{u_{i}}\left(\prod_{j=1}^{n} q_{i j}^{-}\right)^{w_{i}}\right], x=\overline{1, n} .
\end{aligned}
$$


We will do in the expression (4) the change of variables

$$
k_{x}=\alpha_{x}+b_{x}-d_{x}-g_{x}+H-h_{x}-r_{x}+U-u_{x}-w_{x},
$$

then $b_{x}=k_{x}-\alpha_{x}+d_{x}+g_{x}-H+h_{x}+r_{x}-U+u_{x}+w_{x}$ and considering that all network QS function under high load conditions, we obtain

$$
\begin{aligned}
& N_{x}(t)=a_{0}(t) \sum_{\substack{c_{j}=0 \\
j=1, n, j \neq i}}^{\infty} \sum_{\substack{d_{j}=0 \\
j=1, n, j \neq i}}^{\alpha_{j}-g_{j}-h_{j}-r_{j}-u_{j}-w_{j}+H+U-1} \sum_{\substack{g_{j}=0 \\
j=1, n, j \neq i}}^{\infty} \sum_{\substack{h_{j}=0 \\
j=1, n, j \neq i}}^{\infty} \sum_{\substack{r_{j}=0 \\
j=1, n, j \neq i}}^{\infty} \sum_{\substack{u_{j}=0 \\
j=1, n, j \neq i}}^{\infty} \sum_{\substack{w_{j}=0 \\
j=1, n, j \neq i}}^{\infty} \sum_{k_{j}=1}^{\infty} k_{j=1, n} \times \\
& \times \prod_{i=1}^{n}\left[\frac{\left(k_{i}-\alpha_{i}+2 d_{i}+c_{i}+2 g_{i}+2 h_{i}+2 r_{i}+2 u_{i}+2 w_{i}-H-U\right)}{t^{+}} \times\right. \\
& \times\left(\prod_{j=1}^{n} p_{i j}^{+}\right)^{k_{i}-\alpha_{i}+d_{i}+g_{i}-H+h_{i}+r_{i}-U+u_{i}+w_{i}}\left(\lambda_{0 i}^{-}\right)^{c_{i}}\left(\mu_{i}^{+}\right)^{h_{i}+r_{i}}\left(\mu_{i}^{-}\right)^{d_{i}+g_{i}+u_{i}+w_{i}} q_{i 0}^{d_{i}+g_{i}}\left(q_{i i}^{-}\right)^{g_{i}} \\
& \left.\left(\sum_{j=1}^{n} p_{i j}^{-}\right)^{r_{i}}\left(\prod_{j=1}^{n} q_{i j}^{+}\right)^{u_{i}}\left(\prod_{j=1}^{n} q_{i j}^{-}\right)^{w_{i}}\right], x=\overline{1, n} .
\end{aligned}
$$

Similarly, we can find the relation for the average number of nonactivated signals in the system $S_{x}$ :

$$
\begin{gathered}
N_{x}^{s i g n}(t)=a_{0}(t) \sum_{\substack{b_{j}=0 \\
j=1, n, j \neq i}}^{\infty} \sum_{\substack{d_{j}=0 \\
j=1, n, j \neq i}}^{\alpha_{n+j}-u_{j}-w_{j}+R+W-1} \sum_{\substack{g_{j}=0 \\
j=1, n, j \neq i}}^{\infty} \sum_{\substack{h_{j}=0 \\
j=1, n, j \neq i}}^{\infty} \sum_{\substack{r_{j}=0 \\
j=1, n, j \neq i}}^{\infty} \sum_{\substack{u_{j}=0 \\
j=1, n, j \neq i}}^{\infty} \sum_{\substack{w_{j}=0 \\
j=1, n, j \neq i}}^{\infty} \sum_{l_{j}=1}^{\infty} l_{x=1, n} \times \\
\times \prod_{i=1}^{\sum_{i=1}^{n}\left(l_{i}-\alpha_{n+i}+b_{i}+2 d_{i}+g_{i}+h_{i}+r_{i}+2 u_{i}+2 w_{i}-R-W\right)} \times \\
\times\left(\prod_{j=1}^{n} p_{i j}^{+} p^{b_{i}}\left(\lambda_{0 i}^{-}\right)^{y_{i}-\alpha_{n+i}+d_{i}-R+u_{i}-W+w_{i}}\left(l_{i}-\alpha_{i}^{+}\right)^{h_{i}+r_{i}}\left(\mu_{i}^{-}\right)^{d_{i}+g_{i}+u_{i}+w_{i}} q_{i 0}^{d_{i}+g_{i}}\left(q_{i i}^{-}\right)^{g_{i}}\right. \\
\left.\prod_{j=1}^{n} p_{i j}^{-}\right)^{g_{i}}\left(\prod_{j=1}^{n} q_{i j}^{+}\right)^{u_{i}}\left(\prod_{j=1}^{n} q_{i j}^{-}\right)^{w_{i}} \cdot
\end{gathered}
$$

Example. Let the number of QS in QN equal $n=3$. The intensity of the input stream of positive messages and signals $\lambda_{0 i}^{+}$and $\lambda_{0 i}^{-}$are respectively equal to: $\lambda_{01}^{+}=1.8, \lambda_{02}^{+}=1, \lambda_{03}^{+}=2.14, \lambda_{01}^{-}=2, \lambda_{02}^{-}=3, \lambda_{03}^{-}=3.11$. The intensity of service of positive messages and signals in the QS $S_{i}$ set equals respectively: $\mu_{1}^{+}=2$, $\mu_{2}^{+}=4, \mu_{3}^{+}=3, \mu_{1}^{-}=1, \mu_{2}^{-}=0.5, \mu_{3}^{-}=3$. We assume that the transition probability of positive messages $p_{i j}^{+}$has the form: $p_{12}^{+}=1 / 5, p_{13}^{+}=1 / 5, p_{21}^{+}=1 / 6, p_{23}^{+}=1 / 6$, 
$p_{31}^{+}=1 / 7, p_{32}^{+}=1 / 7$; signals transition probability $p_{i j}^{-}$are equal to: $p_{12}^{-}=1 / 4$, $p_{13}^{-}=1 / 4, p_{21}^{-}=1 / 8, p_{23}^{-}=1 / 8, p_{31}^{-}=1 / 9, p_{32}^{-}=1 / 9$; then the probabilities of leaving the network messages to the external environment $p_{i 0}$ will be equal respectively to: $p_{10}=1 / 10, p_{20}=5 / 12, p_{30}=31 / 63$. We also define the probabilities $q_{i j}^{+}$and $q_{i j}^{-}$, when the signal is triggered by a trigger. Let $q_{12}^{+}=1 / 10, q_{13}^{+}=1 / 10$, $q_{21}^{+}=1 / 8, \quad q_{23}^{+}=1 / 8, \quad q_{31}^{+}=1 / 9, \quad q_{32}^{+}=1 / 9 ; \quad q_{12}^{-}=1 / 9, \quad q_{13}^{-}=1 / 9, \quad q_{21}^{-}=1 / 11$, $q_{23}^{-}=1 / 11, q_{31}^{-}=1 / 12, q_{32}^{-}=1 / 12$. The signal is triggered by a negative message that destroys one positive message in the QS $S_{i}$, and leaves the network with probabilities $q_{10}=26 / 45, q_{20}=25 / 44, q_{30}=11 / 18$. In this case $a_{0}(t)=e^{-\frac{289 t}{20}}$.

Find, for example, the probability of state $P(7,7,7,7,7,7, t)$. It is the coefficient of $z_{1}^{7} z_{2}^{7} z_{3}^{7} z_{4}^{7} z_{5}^{7} z_{6}^{7}$ in the expansion of $\Psi_{2 n}(z, t)$ in multiple series (3), so that when power at $z_{i}$ and $z_{n+i}$ must satisfy the relations

$$
\begin{gathered}
\alpha_{i}+b_{i}-d_{i}-g_{i}+H-h_{i}-r_{i}+U-u_{i}-w_{i}=7, \\
\alpha_{n+i}+c_{i}-d_{i}+R-u_{i}+W-w_{i}=7, i=\overline{1,3} .
\end{gathered}
$$

Then, using (3), we obtain

$$
\begin{aligned}
& P(7,7,7,7,7,7, t)= \\
& =e^{-\frac{289 t}{20}} \sum_{\substack{b_{j}=0 \\
j=1,3, j \neq i}}^{\infty} \sum_{\substack{c_{j}=0 \\
j=1,3, j \neq i}}^{\infty} \sum_{\substack{g_{j}=0 \\
j=1,3, j \neq i}}^{\infty} \sum_{\substack{h_{j}=0 \\
j=1,3, j \neq i}}^{\infty} \sum_{\substack{r_{j}=0 \\
j=1,3, j \neq i}}^{\infty} \sum_{\substack{u_{j}=0 \\
j=1,3, j \neq i}}^{\infty} \sum_{\substack{w_{j}=0 \\
j=1,3, j \neq i}}^{\infty} t^{\sum_{i=1}^{3}\left(2 \alpha_{i}-\alpha_{n+i}+3 b_{i}-g_{i}-h_{i}-r_{i}-7+2 H-R+2 U-W\right)} \times \\
& \times \prod_{i=1}^{n}\left[\frac{\left(\lambda_{0 i}^{+}\right)^{b_{i}}\left(\lambda_{0 i}^{-}\right)^{c_{i}}\left(\mu_{i}^{+}\right)^{h_{i}+r_{i}}\left(\mu_{i}^{-}\right)^{\alpha_{i}+b_{i}+H-h_{i}-r_{i}+U-7} q_{i 0}^{\alpha_{i}+b_{i}-h_{i}-r_{i}-u_{i}-w_{i}+H+U-7}\left(q_{i i}^{-}\right)^{g_{i}}}{b_{i} ! c_{i} !\left(\alpha_{i}+b_{i}-g_{i}+H-h_{i}-r_{i}+U-u_{i}-w_{i}-7\right) ! g_{i} ! h_{i} ! r_{i} ! u_{i} ! w_{i} !} \times\right. \\
& \left.\times\left(\prod_{j=1}^{n} p_{i j}^{+}\right)^{h_{i}}\left(\prod_{j=1}^{n} p_{i j}^{-}\right)^{r_{i}}\left(\prod_{j=1}^{n} q_{i j}^{+}\right)^{u_{i}}\left(\prod_{j=1}^{n} q_{i j}^{-}\right)^{w_{i}}\right] .
\end{aligned}
$$

Figure 1 shows plots of the probability of the state $P(7,7,7,7,7,7, t)$ and on condition that at the initial time network is at one of three states: a) $\alpha_{i}=3, i=\overline{1,6}$, b) $\alpha_{i}=1, i=\overline{1,6}$, c) $\alpha_{i}=0, i=\overline{1,6}$.

The average number of messages in network systems (in the queue and in servicing), on condition that $N_{m}(0)=0, m=\overline{1, n}$, can be found by the formula (5), and the average number of signals in the system network (not in the nonactivated state) may be found by the formula (6). 


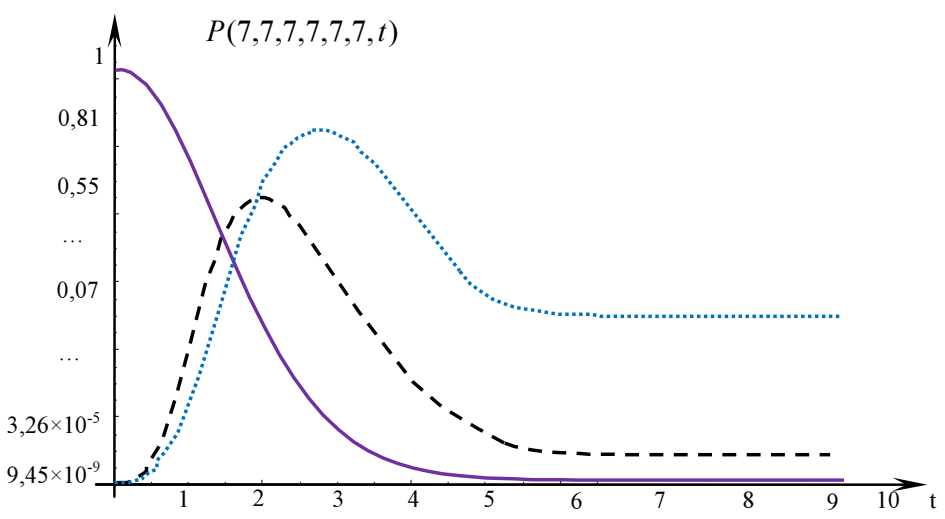

Fig. 1. The chart of the probability of the state $P(7,7,7,7,7,7, t)$

(The continuous line - case a), the dashed - case b), the dotted - c))

Figure 2 shows a graph of change in the average number of messages in the QS $S_{3}$ and a graph of change in the average number of signals in the QS $S_{1}$ respectively.

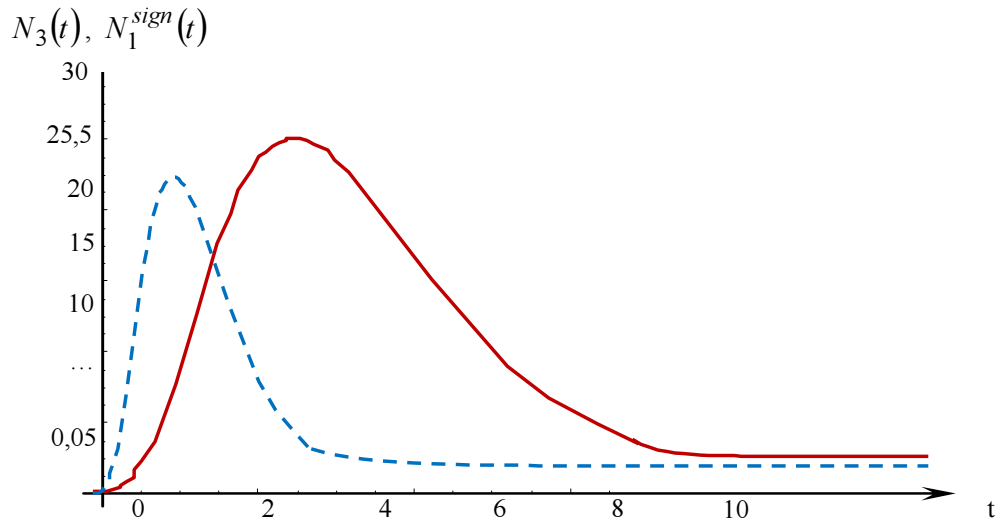

Fig. 2. The average number of messages $N_{3}(t)$ in QS $S_{3}$ and the average number of signals $N_{1}^{\text {sign }}(t)$ in QS $S_{1}$ (the dashed line)

\section{Conclusion}

We propose a method of finding nonstationary state probabilities of the G-network with a one-line QS and also with signals with random delay, based on the use of multivariate generating functions. The proposed approach can serve as a basis for the development of the approximate method of research specified network. Approximate expressions were obtained for time-dependent probabilities of states and the average characteristics of the network, provided that the network is operating under a high load. As described, the application of the results obtained 
on the model computer system attacks and virus penetration effect into a computer network. Further studies in this direction are connected to yield similar results for networks with incomes.

\section{References}

[1] Naumenko V., Matalytski M., Investigation of G-network with signals at transient behavior, Distributed Computer and Communication Networks: Control, Computation, Communications, Proc. of the 17th Intern. Conf. Moscow. M. MSU. 2013, 440-447 (in Russian).

[2] Bocharov P., Queueing network with signals with random delay, Automatics and Telemechanics 2002, 9, 90-101 (in Russian).

[3] Vishnevsky V., Theoretical Bases of Designing Computer Networks, Technosphere, M.: 2003, 512 p. (in Russian).

[4] Matalytski M., Naumenko V., Analysis of the G-network with a random delay of signals in a transient behaviour and its application, Vestnik GrSU, Ser. 2, 2014, 1, 135-147 (in Russian).

[5] Matalytski M., Naumenko V., Nonstationary analysis of queueing network with positive and negative messages, Journal of Applied Mathematics and Computational Mechanics 2013, 12, 2, 61-71.

[6] Faytts F., Dzhnonson P., Kratz M., Computer Virus: Problems and Prognosis, Mir, 1994, 176 p. (in Russian).

[7] Kaspersky LAB Threats. ABCs of security, Directory threats in 2013, Access mode: http://www.kaspersky.ru/threats_faq (Electronic resource).

[8] Kaspersky DDoS Prevention DDoS-attack. 2013, Access mode: http://www.kaspersky.ru/ddosprevention (Electronic resource).

[9] DDoS-attacks 2010, Access mode: http://ddosattack.ru/ (Electronic resource).

[10] SecurityLAB by Positive technologies: DDoS-attacks. 2012, Access mode: http://www.security lab.ru/news/tags/DDoS (Electronic resource). 